Research Article

\title{
Isomorphism Theorems for Groupoids and Some Applications
}

\author{
Jesús Ávila $\left(\mathbb{0},{ }^{1}\right.$ Víctor Marín $\left(\bullet,{ }^{1}\right.$ and Héctor Pinedo ${ }^{2}$ \\ ${ }^{1}$ Departamento de Matemáticas y Estadística, Universidad del Tolima, Santa Helena, Ibagué, Colombia \\ ${ }^{2}$ Escuela de Matemáticas, Universidad Industrial de Santander, Cra. 27 calle 9, Bucaramanga, Colombia \\ Correspondence should be addressed to Jesús Ávila; javila@ut.edu.co
}

Received 4 December 2019; Accepted 27 January 2020; Published 26 February 2020

Academic Editor: Luca Vitagliano

Copyright @ 2020 Jesús Ávila et al. This is an open access article distributed under the Creative Commons Attribution License, which permits unrestricted use, distribution, and reproduction in any medium, provided the original work is properly cited.

Using an algebraic point of view we present an introduction to the groupoid theory; that is, we give fundamental properties of groupoids as uniqueness of inverses and properties of the identities and study subgroupoids, wide subgroupoids, and normal subgroupoids. We also present the isomorphism theorems for groupoids and their applications and obtain the corresponding version of the Zassenhaus Lemma and the Jordan-Hölder theorem for groupoids. Finally, inspired by the Ehresmann-ScheinNambooripad theorem we improve a result of R. Exel concerning a one-to-one correspondence between partial actions of groups and actions of inverse semigroups.

\section{Introduction}

The concept of groupoid from an algebraic point of view appeared for the first time in [1]. From this setting, a (Brandt) groupoid can be seen as a generalization of a group, that is, a set with partial multiplication on it that could contain many identities.

Brandt groupoids were generalized by Ehresmann in [2], where the author added further structures such as topological and differentiable structures. Other equivalent definitions of groupoids and their properties are given in [3], where a groupoid is defined as a small category where each morphism is invertible.

In Definition 1.1 of [4], the author follows the definition given by Ehresmann and presents the notion of groupoid as a particular case of universal algebra, and he defines strong homomorphism for groupoids and proves the correspondence theorem in this context. The Cayley theorem for groupoids is also presented in Theorem 3.1 of [5].

Recently, some applications of groupoids to the study of partial actions are presented in different branches, for instance, in [6] the author constructs a Birget-Rhodes expansion $\mathscr{G}^{\mathrm{BR}}$ associated with an ordered groupoid $\mathscr{G}$ and shows that it classifies partial actions of $\mathscr{G}$ on sets, in the topological context in [7] is treated the globalization problem, connections between partial actions of groups and groupoids are given in $[8,9]$. Also, ring theoretic and cohomological results of global and partial actions of groupoids on algebras are obtained in [10-16]. Galois theoretic results for groupoid actions are obtained in [12, 17-19]. Finally, the globalization problem for partial groupoid actions has been considered in $[7,20,21]$.

In [19], Paques and Tamusiunas give some structural definitions in the context of groupoid such as abelian groupoid, subgroupoid, and normal subgroupoid and showed necessary and sufficient conditions for a subgroupoid to be normal. Furthermore, they built quotient groupoids.

Due to the applications of the groupoids to partial actions and their usefulness, we will give an elementary introduction to the theory of groupoids from an axiomatic definition following Lawson [22].

Our principal goal in this work is to continue the algebraic development of a groupoid theory. The paper is organized as follows. After the introduction, in Section 2, we present groupoids from an axiomatic point and show some properties of them. In Section 3 we recall the notions of some substructures of groupoids, such as subgroupoid, wide subgroupoid, and normal subgroupoid. In Section 4, we prove the correspondence and isomorphism theorems for 
groupoids. In the final section we show an application of section four, we prove the Zassenhaus Lemma and Hölder theorem for groupoids, and we improve Theorem 4.2 of [23] using the Ehresmann-Schein-Nambooripad theorem.

It is important to note that the notion of the groupoid can be presented from categories, algebraic structures, and universal algebra. In the last setting, the isomorphism theorems are valid, but the idea is to do an algebraic presentation and verify which assumptions are necessary. So it is possible to reach a wider audience.

\section{Groupoids}

Now, we give two definitions of groupoids from an algebraic point of view.

Definition 1 (see [22], p. 78). Let $\mathscr{G}$ be a set equipped with a partial binary operation on $\mathscr{G} \times \mathscr{G}$ which is denoted by concatenation. If $g, h \in \mathscr{G}$ and the product $g h$ is defined, we write $\exists g h$. An element $e \in \mathscr{G}$ is called an identity if

$$
\begin{gathered}
\exists e g \text { implies } e g=g, \\
\exists g^{\prime} e \text { implies } g^{\prime} e=g^{\prime} .
\end{gathered}
$$

The set of identities of $\mathscr{G}$ is denoted by $\mathscr{G}_{0}$. Then $\mathscr{G}$ is said to be a groupoid if the following axioms hold:

(i) $\exists g(h l)$, if and only if $\exists(g h) l$ and $g(h l)=(g h) l$

(ii) $\exists g(h l)$, if and only if $\exists g h$ and $\exists h l$

(iii) For each $g \in \mathscr{G}$, there are unique identities $d(g)$ and $r(g)$ such that $\exists g d(g)$ and $\exists r(g) g$

(iv) For each $g \in \mathscr{G}$, there is an element $g^{-1} \in \mathscr{G}$ such that $\exists g^{-1} g, \exists g g^{-1}, d(g)=g^{-1} g$, and $r(g)=g g^{-1}$

The following definition of groupoid is presented in Definition 1.1 of [24].

Definition 2. A groupoid is a set endowed with a product map

$$
\mathscr{G}^{2} \exists(g, h) \longmapsto g h \in \mathscr{G},
$$

where the set $\mathscr{G}^{2} \subseteq \mathscr{G} \times \mathscr{G}$ is called the set of composible pairs and an inverse map $\mathscr{G} \ni g \longmapsto g^{-1} \in \mathscr{G}$ such that for all $g, h, l \in \mathscr{G}$ the following relations are satisfied.

$$
\begin{aligned}
& \text { (G1) }\left(g^{-1}\right)^{-1}=g \\
& \text { (G2) If }(g, h),(h, l) \in G^{2}, \text { then }(g h, l),(g, h l) \in \mathscr{G}^{2} \text { and } \\
& \quad(g h) l=g(h l) \\
& \text { (G3) }\left(g^{-1}, g\right) \in \mathscr{G}^{2} \text { and if }(g, h) \in \mathscr{G}^{2} \text {, then } g^{-1}(g h)=h \\
& \text { (G4) }\left(g, g^{-1}\right) \in \mathscr{G}^{2} \text { and if }(l, g) \in \mathscr{G}^{2}, \text { then }(l g) g^{-1}=l
\end{aligned}
$$

We shall check that Definitions 1 and 2 are equivalent. First, we need a couple of lemmas.

Lemma 1 (see [25], Lemma 1.1.4). Suppose that $\mathscr{G}$ is a groupoid in the sense of Definition 1. Let $g, h \in \mathscr{G}$. Then $\exists g h$, if and only if $d(g)=r(h)$.
Proof. Let $g, h \in \mathscr{G}$ such that $\exists g h$. By (iv) of Definition 1, we have that $\exists g^{-1}, \exists h^{-1}, d(g)=g^{-1} g$, and $r(h)=h h^{-1}$. Since $\exists g h$, then $\exists g^{-1} g h h^{-1}$. That is, $\exists d(g) r(h)$. Now, since $d(g)$ and $r(h)$ are identities, then $d(g)=d(g) r(h)=r(h)$. Conversely, if $d(g)=r(h)$, then $\exists \operatorname{gr}(h)$, and since $\operatorname{gr}(h)=$ $g\left(h h^{-1}\right)$ we have $\exists g\left(h h^{-1}\right)$. Whence by (ii) of Definition 1, we have that $\exists g h$.

Lemma 2. Suppose that $\mathscr{G}$ is a groupoid in the sense of Definition 1. Then, the element $g^{-1}$ in (iv) is unique and $\left(g^{-1}\right)^{-1}=g$.

Proof. For each $g \in \mathscr{G}$, assume that there exists $y, z \in \mathscr{G}$ such that $\exists y g, \exists g y, \exists z g, \exists g z, y g=d(g)=z g$, and $g y=$ $r(g)=g z$. Notice that $y g=z g$ which implies that $(y g) g^{-1}=(z g) g^{-1}$, which is defined by (ii) of Definition 1, and then by associativity, $y r(g)=z r(g)$. Thus, $y d(y)=$ $z d(z)$, so $y=z$. It is analogous for $g y=g z$. In particular, the inverse is unique.

Finally, the equality $\left(g^{-1}\right)^{-1}=g$ follows from the uniqueness of the inverse of $g^{-1}$.

We give the following.

Proposition 1. Let $\mathscr{G}$ be a set. Then, it is a groupoid in the sense of Definition 1, if and only if it is a groupoid in the sense of Definition 2.

Proof. Let $\mathscr{G}^{2}=\{(g, h) \in \mathscr{G} \times \mathscr{G} \mid \exists g h\}$. By using (iv) of Definition 1, we define $\mathscr{G} \ni g \longmapsto g^{-1} \in \mathscr{G}$. Then, by Lemma 2 this map is well defined. We shall check (G1)-(G4) of Definition 2:

(G1) It is the second assumption in Lemma 2.

(G2) If $(g, h),(h, l) \in \mathscr{G}^{2}$, then $\exists g h$ and $\exists h l$. By (i) and (ii), $\exists(g h) l$ and $\exists g(h l)$, that means $(g h, l)$, $(g, h l) \in \mathscr{G}^{2}$ and $(g h) l=g(h l)$.

(G3) By item (iv), we get that $\left(g^{-1}, g\right) \in \mathscr{G}^{2}$. Let $h \in \mathscr{G}$ with $(g, h) \in \mathscr{G}^{2}$. By Lemma 1 , we get that $d(g)=$ $r(h)$, and by using (iii), we obtain $g^{-1}(g h)=h$.

(G4) This is proved analogously to the previous item.

Conversely, suppose that $\mathscr{G}$ is a set. We define a partial binary operation on $\mathscr{G}$ by $\exists g h$, if and only if $(g, h) \in \mathscr{G}^{2}$ and $g h=m(g, h)$. We shall check that properties (i)-(iv) in Definition 1 hold:

(i) Let $g, h, l \in \mathscr{G}$ such that $\exists g(h l)$. Then $(g, h l),(h, l) \in \mathscr{G}^{2}$ and by (G4), $\left(l, l^{-1}\right) \in \mathscr{G}^{2}$ and $h=$ $(h l) l^{-1}$. Thus, $(g, h l),\left(h l, l^{-1}\right) \in \mathscr{G}^{2}$ and by (G2), $\left(g(h l), l^{-1}\right) \in \mathscr{G}^{2}$ and $[g(h l)] l^{-1}=g\left[(h l) l^{-1}\right]=g h$. In particular, $(g, h) \in \mathscr{G}^{2}$. We conclude that $(g, h),(h, l) \in \mathscr{G}^{2}$ and by using (G2), we get that $(g h) l=g(h l)$. Conversely, suppose that $\exists(g h) l$. Then, $(g, h),(g h, l) \in \mathscr{G}^{2}$ and by (G3), we have that $\left(g^{-1}, g\right) \in \mathscr{G}^{2}$ and $g^{-1}(g h)=h$. Thus, $\left(g^{-1}, g h\right),(g h, l) \in \mathscr{G}^{2}$ and by $(\mathrm{G} 2), \quad(h, l) \in \mathscr{G}^{2}$. Finally, since $(g, h) \in \mathscr{G}^{2}$ we obtain, again by (G2), that $(g, h l) \in \mathscr{G}^{2}$. Hence, $\exists g(h l)$.

(ii) This is shown analogously to the previous items. 
(iii) (iv) If $g \in \mathscr{G}$, then $\left(g^{-1}, g\right),\left(g, g^{-1}\right) \in \mathscr{G}^{2}$. Thus, we set $r(g)=g g^{-1}$ and $d(g)=g^{-1} g$. Hence, by (G2), (G3), and (G4), $\exists g d(g), \exists d(g) g$, and the equalities $g d(g)=g=r(g)(g)$ hold.

Remark 1. The interested reader can find another two equivalent definitions of groupoids in [26, 27].

From now on in this work $\mathscr{G}$ denotes a groupoid.

For the sake of completeness, we give the proof of some known consequences of Definition 1.

Proposition 2 (see [25], Lemma 1.1.4). For each $g, h, k, l \in \mathscr{G}$ we have:

(i) If $\exists g h$, then $d(g h)=d(h)$ and $r(g h)=r(g)$.

(ii) $\exists g h$, if and only if $\exists h^{-1} g^{-1}$, and in this case $(g h)^{-1}=$ $h^{-1} g^{-1}$.
Proof

(i) For the first equality, we prove that $d(h)$ satisfies the axiom (iii) from Definition 1. Indeed, assume that $\exists g h$. Then, $\exists(g h) d(h), d(g)=r(h)$, and

$$
(g h) d(h)=g(h d(h))=g h .
$$

In a similar way, it is possible to show that $r(g h)=r(g)$

(ii) We have that $\exists g h$, if and only if $d(g)=r(h)$. Notice that for any $l \in \mathscr{G}$ we have that

$$
d(l)=l^{-1} l=l^{-1}\left(l^{-1}\right)^{-1}=r\left(l^{-1}\right) .
$$

Then, $d\left(h^{-1}\right)=r\left(g^{-1}\right)$. That is, $\exists h^{-1} g^{-1}$. Furthermore,

$$
\begin{aligned}
& g h\left(h^{-1} g^{-1}\right)=g\left(h h^{-1}\right) g^{-1}=g r(h) g^{-1}=g d(g) g^{-1}=g g^{-1}=r(g)=r(g h), \\
& \left(h^{-1} g^{-1}\right) g h=h^{-1}\left(g^{-1} g\right) h=h^{-1} d(g) h=h^{-1} r(h) h=h^{-1} h=d(h)=d(g h) .
\end{aligned}
$$

Therefore, by the uniqueness of the inverse element, we get that $(g h)^{-1}=h^{-1} g^{-1}$.

The following statements also follow from the definition of groupoid.

Proposition 3. Let $g \in \mathscr{G}$. Then, the following statements hold:

$$
\begin{aligned}
& \text { (i) } d(g)=r\left(g^{-1}\right), \\
& \text { (ii) } d(d(g))=d(g), \\
& \text { (iii) } r(r(g))=r(g), \\
& \text { (iv) } d(r(g))=r(g), \\
& \text { (v) } r(d(g))=d(g) .
\end{aligned}
$$

Proof

(i) This is (4)

(ii) $d(d(g))=d\left(g^{-1} g\right)=d(g)$, where the last equality follows from (i) of Proposition 2

(iii) $r(r(g))=r\left(g g^{-1}\right)=r(g)$, where the last equality also follows from (i) of Proposition 2

Items (iv) and (v) are proved analogously.

Remark 2. Let $\mathscr{G}$ be a groupoid. In ([12], p. 3660), Bagio and Paques called an element $e \in \mathscr{G}$ an identity if $e=d(g)$, for some $g \in \mathscr{G}$.

Proposition 4. Let $\mathscr{G}$ be a groupoid. An element ef $\mathscr{G}$ is an identity in the sense of Bagio and Paques, if and only if it satisfies (1).
Proof. Suppose that $e=d(h)$ is an identity in the sense of Bagio and Paques, for some $h \in \mathscr{G}$. By (i) of Proposition 3, $e=d(h)=r\left(h^{-1}\right)$. Now, let $g, g^{\prime} \in \mathscr{G}$ such that $\exists e g$ and $\exists g^{\prime} e$. By Lemma 1 and (ii)-(v) of Proposition 3, we have that $d\left(g^{\prime}\right)=r(e)=e=d(e)=r(g)$, then $e g=r(g) g=g$ and $g^{\prime} e=g^{\prime} d\left(g^{\prime}\right)=e$. Therefore, $e$ satisfies (1).

Conversely, suppose that $e \in \mathscr{G}$ satisfies (1). By (iii) of Definition 1, we get $\exists e d(e)$ and $e=e d(e)$. Thus $e=d(e)$, and it follows that $e$ is an identity in the sense of Bagio and Paques.

Remark 3. It follows from the proof of Proposition 4 that $d(e)=e=r(e), \exists e e$, and $e e=e=e^{-1}$ for any $e \in \mathscr{G}_{0}$. Moreover, note that the elements of $\mathscr{G}_{0}$ are the unique idempotents of $\mathscr{G}$. In fact, if $\exists g^{2}$ and $g^{2}=g$, then $(g g) g^{-1}=$ $g g^{-1}$ and so $g r(g)=g g^{-1}=r(g)$. Since $d(g)=r(g)$, it follows that $g=g d(g)=g r(g)=r(g)$.

Proposition 5. Let $e \in \mathscr{G}_{0}$. Then, the set $\mathscr{G}_{e}=$ $\{g \in G \mid d(g)=r(g)=e\}$ is a group.

Proof. By Remark 3, we have that $d(e)=r(e)=e$. Thus $e \in \mathscr{G}_{e}$. If $g, h \in \mathscr{G}_{e}$, then $d(g)=e=r(h)$, and so $\exists g h$ thanks to Lemma 1. Now, (i) of Proposition 2 implies that $d(g h)=$ $d(h)=e$ and $r(g h)=r(g)=e$. Hence, $g h \in \mathscr{G}_{e}$. If $g \in \mathscr{G}_{e}$, then by Lemma 1, $\exists g e$ and $\exists e g$ and we have that $g e=$ $g d(g)=g$ and $e g=r(g) g=g$. Therefore, $e$ is the identity element of $\mathscr{G}_{e}$. Finally, let $g \in \mathscr{G}_{e}$. By Proposition 3, $d\left(g^{-1}\right)=r(g)=e$ and $r\left(g^{-1}\right)=d(g)=e$. Hence, $g^{-1} \in \mathscr{G}_{e}$, $g g^{-1}=g^{-1} g=e$, and we conclude that $\mathscr{G}_{e}$ is a group.

Definition 3. The group $\mathscr{G}_{e}$ is called the isotropy group associated with $e$. The isotropy subgroupoid (see Definition 5) or the group bundle associated to $\mathscr{G}$ is defined by the disjoint union Iso $(\mathscr{G})=\cup_{e \in \mathscr{G}_{0}} \mathscr{G}_{e}$. 
Remark 4. A concept of abelian groupoid was presented in ([19], p. 111) as follows: a groupoid $\mathscr{G}$ is abelian if $d(g)=$ $r(g)$ for each $g \in \mathscr{G}$; and $g h=h g$, for all $g, h \in \mathscr{G}$ with $d(g)=r(h)$.

We have the following.

Proposition 6. A groupoid $\mathscr{G}$ is abelian in the sense of Paques and Tamusiunas, if and only if $\mathscr{G}=I s o(\mathscr{G})$ and $\mathscr{G}_{e}$ is abelian for all $e \in \mathscr{G}_{0}$.

In the light of Proposition 6, we prefer to use the following definition of the abelian groupoid.

Definition 4 (see [28], Definition 1.1). A groupoid $\mathscr{G}$ is called abelian if all its isotropy groups are abelian.

Note that if $\mathscr{G}$ is abelian in the sense of Paques and Tamusiunas, then it is abelian in the sense of Definition 4. Now, consider the groupoid $\mathscr{G}=\left\{g, g^{-1}, d(g), r(g)\right\}$ with $d(g) \neq r(g)$. Then, we have that $\mathscr{G}_{d(g)}=\{d(g)\}$ and $\mathscr{G}_{r(g)}=\{r(g)\}$. That is, $\mathscr{G}$ is an abelian groupoid in the sense of Definition 4, but it is not a union of abelian groups.

\section{Normal Subgroupoids, the Quotient Groupoid and Homomorphisms}

In this section, we present a theory of substructures in a groupoid. We follow the definition of subgroupoid given in [19].

Definition 5. Let $\mathscr{G}$ be a groupoid and $\mathscr{H}$ a nonempty subset of $\mathscr{G} . \mathscr{H}$ is said to be a subgroupoid of $\mathscr{G}$ if it satisfies: for all $g, h \in \mathscr{H}$,

(i) $g^{-1} \in \mathscr{H}$

(ii) If $\exists g h$, then $g h \in \mathscr{H}$

If $\mathscr{H}$ is a subgroupoid of $\mathscr{G}$, then it is called wide if $\mathscr{H}_{0}=\mathscr{G}_{0}$.

Remark 5. It is clear that if $\mathscr{H}$ is a subgroupoid of $\mathscr{G}$, then it is a groupoid with the product (2), restricted to $\mathscr{H}^{2}=(\mathscr{H} \times \mathscr{H}) \cap \mathscr{G}^{2}$.

\section{Example 1. Let $\mathscr{G}$ be a groupoid.}

(1) Take $a \in \mathscr{G}$ such that $d(a)=r(a)$. The set $C(a)=$ $\left\{g \in \mathscr{G}_{d(a)} \mid g a=a g\right\}$ is a subgroupoid of $\mathscr{G}$. Indeed, first of all note that by assumption $a \in C(a)$. If $x, y \in C(a)$, then $x, y \in \mathscr{G}_{d(a)}, \quad x a=a x$, and $y a=a y$. Since $\mathscr{G}_{d(a)}$ is a group, then $\exists(x y) a$ and

$$
\begin{aligned}
(x y) a & =x(y a)=x(a y)=(x a) y \\
& =(a x) y=a(x y) .
\end{aligned}
$$

That is, $x y \in C(a)$. If $x \in C(a)$, then $x^{-1} \in \mathscr{G}_{d(a)}$. Hence, we have that $\exists x^{-1} a$ and $x^{-1} a=a x^{-1}$ since $a x=x a$. Observe that this example generalizes the concept of centralizer in groups.
(2) Suppose that $\mathscr{G}$ is abelian and $n>1$. Then the set $\mathscr{H}_{n}=\left\{a^{n} \mid a \in \operatorname{Iso}(\mathscr{G})\right\}$ is a subgroupoid of $\mathscr{G}$. If $x, y \in \mathscr{H}_{n}$, then $x=a^{n}, y=b^{n}$ for some $a, b \in \operatorname{Iso}(\mathscr{G})$. If $\exists x y$ then $\exists a^{n} b^{n}$, and this implies that $\exists a b$ and thus $a, b \in \mathscr{G}_{e}$ for some $e \in \mathscr{G}_{0}$. Then, $a b=b a$, and so $x y=(a b)^{n} \in \mathscr{H}$. Now, if $x \in \mathscr{H}$, then $\quad x=a^{n}$ for some $a \in \mathscr{G}$. Thus, $x^{-1}=\left(a^{n}\right)^{-1}=\left(a^{-1}\right)^{n} \in \mathscr{H}_{n}$. Finally, note that for $g \in \mathscr{G}, d(g)=d(g)^{n} \in \mathscr{H}_{n}$. Hence, $\mathscr{G}_{0} \subseteq \mathscr{H}$, and we conclude that $\mathscr{H}$ is wide.

(3) Suppose that $\mathscr{G}$ is abelian. Then, the set $\operatorname{Tor}(\mathscr{G})=$ $\left\{g \in \operatorname{Iso}(\mathscr{G}) \mid g^{n} \in \mathscr{G}_{0}\right.$ for some $\left.n \in \mathbb{N}\right\}$ is a wide subgroupoid of $\mathscr{G}$. First, it is clear that $\mathscr{G}_{0} \subseteq \operatorname{Tor}(\mathscr{G})$. If $g, h \in \operatorname{Tor}(\mathscr{G})$, then $g^{n}=e, h^{k}=f$ for some $n, k \in \mathbb{N}$ and some $e, f \in \mathscr{G}_{0}$. Thus, we obtain that $d(g)=d\left(g^{n}\right)=e=r\left(g^{n}\right)=r(g)$ and $d(h)=d\left(h^{k}\right)=f=r\left(h^{k}\right)=r(h)$. If $\exists g h$, then $d(g)=r(h)$ and thus $e=f$ and $g h=h g$, since $\mathscr{G}$ is an abelian groupoid. Then $(g h)^{n k}=g^{n k} h^{n k}=\left(g^{n}\right)^{k}\left(h^{k}\right)^{n}=d(g)$, that is, $g h \in \operatorname{Tor}(\mathscr{G})$. Now, since $g^{n}=e$ we have $\left(g^{-1}\right)^{n}=\left(g^{n}\right)^{-1}=e$, and hence $g^{-1} \in \operatorname{Tor}(\mathscr{G})$. We conclude that $\operatorname{Tor}(\mathscr{G})$ is a wide subgroupoid of $\mathscr{G}$. Note that if we take a fixed $n \in \mathbb{N}$ and define the set $\mathscr{D}_{n}=\left\{g \in \mathscr{G} \mid g^{n} \in \mathscr{G}_{0}\right\}$, then $\mathscr{D}_{n}$ is a wide subgroupoid of $\mathscr{G}$ and $\mathscr{D}_{n} \subseteq \operatorname{Tor}(\mathscr{G})$. That is, $\mathscr{D}_{n}$ is a subgroupoid of Tor $(\mathscr{G})$. Observe that this example generalizes the concept of torsion subgroup in abelian groups.

Proposition 7. Let $\mathscr{G}$ be a groupoid and $\mathscr{H}, \mathscr{K}$ subgroupoids of $\mathscr{G}$. Then,

(i) If $\mathscr{H} \mathscr{K}$ is non-empty, then $\mathscr{H} \mathscr{K}$ is a subgroupoid of $\mathscr{G}$, if and only if $\mathscr{H} \mathscr{K}=\mathscr{K} \mathscr{H}$

(ii) If $\mathscr{H}$ and $\mathscr{K}$ are wide and $\mathscr{H} \mathscr{K}$ is a subgroupoid, then $\mathscr{H} \mathscr{K}$ is wide

Proof. The proof of (i) is similar to the group case. To prove (ii), it is enough to observe that $\mathscr{G}_{0}=\mathscr{H}_{0}=\mathscr{K}_{0}$ and if $e \in \mathscr{G}_{0}$, then $e=e e \in \mathscr{H} \mathscr{K}$.

Now, we present the notion of a normal subgroupoid and prove several properties of them, which generalize wellknown results in group theory. We follow the definition given in [19].

Definition 6. Let $\mathscr{G}$ be a groupoid. The subgroupoid $\mathscr{H}$ of $\mathscr{G}$ is said to be normal, denoted by $\mathscr{H} \triangleleft \mathscr{G}$, if $g^{-1} \mathscr{H} g \neq \varnothing$ and $g^{-1} \mathscr{H} g \subseteq \mathscr{H}$, for all $g \in \mathscr{G}$, where $g^{-1} \mathscr{H} g=\left\{g^{-1} h g \mid h \epsilon\right.$ $\left.\mathscr{H} \cap \mathscr{G}_{r(g)}\right\}$.

Remark 6. By the proof of Lemma 3.1 of [19], one has that $g^{-1} \mathscr{H} g \neq \varnothing$, if and only if $\mathscr{H}$ is wide. Also the assertion $g^{-1} \mathscr{H} g \subseteq \mathscr{H}$ is equivalent to $g^{-1} \mathscr{H}_{r(g)} g=\mathscr{H}_{d(g)}$, for all $g \in \mathscr{G}$.

Several examples of normal groupoids are presented in [19], p. 110-111. 
Given a wide subgroupoid $\mathscr{H}$ of $\mathscr{G}$, in [19], Paques and Tamusiunas define a relation on $\mathscr{G}$ as follows: for every $g, l \in \mathscr{G}$

$$
g \equiv \mathscr{H} l \Longleftrightarrow\left(\exists l^{-1} g \text { and } l^{-1} g \in \mathscr{H}\right) .
$$

Furthermore, they prove that this relation is a congruence, which is an equivalence relation that is compatible with products. The equivalence class of $\equiv_{\mathscr{H}}$ containing $g$ is the set $g \mathscr{H}=\{g h \mid h \in \mathscr{H} \wedge r(h)=d(g)\}$. This set is called the left coset of $\mathscr{H}$ in $\mathscr{G}$ containing $g$. Then, we have the following.

Proposition 8 (see [19], Lemma 3.12). Let $\mathscr{H}$ be a normal subgroupoid of $\mathscr{G}$ and let $\mathscr{G} / \mathscr{H}$ be the set of all left cosets of $\mathscr{H}$ in $\mathscr{G}$. Then, $\mathscr{G} / \mathscr{H}$ is a groupoid such that $\exists(g \mathscr{H})(l \mathscr{H})$, if and only if $\exists g l$ and the partial binary operation is given by $(g \mathscr{H})(l \mathscr{H})=g l \mathscr{H}$.

The groupoid $\mathscr{G} / \mathscr{H}$ in Proposition 8 is called the quotient groupoid of $\mathscr{G}$ by $\mathscr{H}$.

Now, we present the notion of groupoid homomorphism and prove several properties of them, which generalize wellknown results in homomorphisms of groups.

Definition 7. Let $\mathscr{G}$ and $\mathscr{G}^{\prime}$ be groupoids. A map $\phi: \mathscr{G} \longrightarrow \mathscr{G}^{\prime}$ is called groupoid homomorphism if for all $x, y \in \mathscr{G}, \exists x y$ implies that $\exists \phi(x) \phi(y)$, and in this case $\phi(x y)=\phi(x) \phi(y)$.

Notice that $j: \mathscr{G} \longrightarrow \mathscr{G} / \mathscr{H}$ defined by $g \longmapsto g H$ for all $g \in \mathscr{G}$, is a surjective groupoid homomorphism.

Definition 8. Let $\phi: \mathscr{G} \longrightarrow \mathscr{G}^{\prime}$ be a homomorphism of groupoids. We define the following sets:

(i) For $\mathscr{H} \subseteq \mathscr{G}$, write $\phi(\mathscr{H})=\left\{\phi(h) \in \mathscr{G}^{\prime} \mid h \in \mathscr{H}\right\}$, the direct image of $\mathscr{H}$. In particular, the set $\phi(\mathscr{G})$ is called the image of $\phi$.

(ii) $\operatorname{Ker}(\phi)=\left\{g \in \mathscr{G} \mid \phi(g) \in \mathscr{G}_{0}^{\prime}\right\}$, the kernel of $\phi$.

(iii) Let $\mathscr{H}^{\prime} \subseteq \mathscr{G}^{\prime}, \phi^{-1}\left(\mathscr{H}^{\prime}\right)=\left\{g \in \mathscr{G} \mid \phi(g) \in \mathscr{H}^{\prime}\right\}$, the inverse image of $\mathscr{H}^{\prime}$ by $\phi$.

(iv) $\phi$ is called a monomorphism if it is injective, an epimorphism if it is surjective, and an isomorphism if it is bijective.

Remark 7. If $\mathscr{G}$ is abelian and $\mathscr{H}$ is a subgroupoid of $\mathscr{G}$, then it is not difficult to show that $\mathscr{H}$ is abelian. Moreover, if $\mathscr{G}^{\prime}$ is another groupoid, such that there is a groupoid epimorphism $\phi: \mathscr{G} \longrightarrow \mathscr{G}^{\prime}$, then $\mathscr{G}^{\prime}$ is also abelian.

Proposition 9. Let $\phi: \mathscr{G} \longrightarrow \mathscr{G}^{\prime}$ be a groupoid homomorphism. Then,

(i) For each $a \in \mathscr{G}, \phi(d(a))=d(\phi(a)), \quad \phi(r(a))=$ $r(\phi(a))$ and $\phi\left(a^{-1}\right)=(\phi(a))^{-1}$.

(ii) If $\mathscr{H}^{\prime}$ is a subgroupoid of $\mathscr{G}^{\prime}$, then $\phi^{-1}\left(\mathscr{H}^{\prime}\right)$ is a subgroupoid of $\mathscr{G}$. Moreover, if $\mathscr{H}^{\prime}$ is wide then $\phi^{-1}\left(\mathscr{H}^{\prime}\right)$ is wide, and it contains $\operatorname{Ker}(\phi)$. (iii) If $\mathscr{H}^{\prime} \triangleleft \mathscr{G}^{\prime}$, then $\phi^{-1}\left(\mathscr{H}^{\prime}\right) \triangleleft \mathscr{G}$ and $\operatorname{Ker}(\phi) \subseteq \phi^{-1}\left(\mathscr{H}^{\prime}\right)$. In particular, $\operatorname{Ker}(\phi) \triangleleft \mathscr{G}$.

Proof

(i) Let $a \in \mathscr{G}$. Since $\exists a d(a)$ then $\exists \phi(a) \phi(d(a))$ and $\phi(a)=\phi(a d(a))=\phi(a) \phi(d(a))$. Thus, by the uniqueness of the identities $\phi(d(a))=d(\phi(a))$. Analogously, $\phi(r(a))=r(\phi(a))$. Finally, since $\exists a a^{-1}$ and $\exists a^{-1} a$, then $\exists \phi(a) \phi\left(a^{-1}\right)$ and $\exists \phi\left(a^{-1}\right) \phi(a)$. Moreover,

$$
\begin{aligned}
& \phi(a) \phi\left(a^{-1}\right)=\phi\left(a a^{-1}\right)=\phi(r(a))=r(\phi(a)), \\
& \phi\left(a^{-1}\right) \phi(a)=\phi\left(a^{-1} a\right)=\phi(d(a))=d(\phi(a)),
\end{aligned}
$$

Which implies that $\phi\left(a^{-1}\right)=(\phi(a))^{-1}$.

(ii) It is not difficult to show that $\phi^{-1}\left(\mathscr{H}^{\prime}\right)$ is a subgroupoid of $\mathscr{G}$. Now suppose that $\mathscr{H}^{\prime}$ is wide. By item (i), we know that $\phi\left(\mathscr{G}_{0}\right) \subseteq \mathscr{G}_{0}^{\prime} \subseteq \mathscr{H}^{\prime}$, that is, $\mathscr{G}_{0} \subseteq \phi^{-1}\left(\mathscr{H}^{\prime}\right)$. Finally, if $x \in \operatorname{Ker}(\phi)$, then $\phi(x) \in \mathscr{G}_{0}^{\prime} \subseteq \mathscr{H}^{\prime}$ and hence $x \in \phi^{-1}\left(\mathscr{H}^{\prime}\right)$, as desired.

(iii) By item (ii), it is enough to see that $g^{-1} \phi^{-1}\left(\mathscr{H}^{\prime}\right) g \subseteq \phi^{-1}\left(\mathscr{H}^{\prime}\right)$ for all $g \in \mathscr{G}$. Indeed, let $g^{-1} l g \in g^{-1} \phi^{-1}\left(\mathscr{H}^{\prime}\right) g$ with $l \in \phi^{-1}\left(\mathscr{H}^{\prime}\right)$ and $d(l)=r(l)=r(g)$. Then, $\quad \exists l g$ and thus $\phi(d(l))=\phi(r(g))$. We have

$$
d\left(\phi\left(g^{-1}\right) \phi(l)\right)=d(\phi(l))=r(\phi(g))
$$

Then, $\exists\left(\phi\left(g^{-1}\right) \phi(l)\right) \phi(g)$, and since $\phi(l) \in \mathscr{H}^{\prime}$ and $\mathscr{H}^{\prime} \triangleleft \mathscr{G}^{\prime}$ we obtain that

$$
\phi\left(g^{-1} l g\right)=\phi\left(g^{-1}\right) \phi(l) \phi(g)=\phi(g)^{-1} \phi(l) \phi(g) \in \mathscr{H}^{\prime} .
$$

Finally, to show that $\operatorname{Ker}(\phi) \triangleleft \mathscr{G}$, it is enough to observe that $\phi^{-1}\left(\mathscr{G}_{0}^{\prime}\right)=\operatorname{Ker}(\phi)$ and $\mathscr{G}_{0}^{\prime}$ is normal in $\mathscr{G}^{\prime}$.

3.1. Strong Isomorphism Theorems for Groupoids. In this section, we present a special type of groupoid homomorphism, called the strong groupoid homomorphism. Using these homomorphisms, we show the correspondence theorem and the isomorphism theorems for groupoids. This notion of strong groupoid homomorphism has been considered before by several authors (see [4], Remark 2.2).

Definition 9. Let $\phi: \mathscr{G} \longrightarrow \mathscr{G}^{\prime}$ be a groupoid homomorphism. $\phi$ is called strong if for all $x, y \in \mathscr{G}, \exists \phi(x) \phi(y)$ implies that $\exists x y$.

Example 2. Let $X$ be a nonempty set and $X^{2}=X \times X$. Then $X^{2}$ is a groupoid, where the product is given by: $(y, z)(x, y)=(x, z)$, for $x, y, z \in X$. Then, the map $f: \mathscr{G} \ni g \longmapsto(d(g), r(g)) \in \mathscr{G}_{0}^{2}$ is a strong groupoid homomorphism with kernel Iso $(\mathscr{G})$. 
Proposition 10. Let $\phi: \mathscr{G} \longrightarrow \mathscr{G}^{\prime}$ be a strong groupoid homomorphism. Then,

(i) If $\mathscr{H}<\mathscr{G}$, then $\phi(\mathscr{H})<\mathscr{G}^{\prime}$, and $\operatorname{Ker}(\phi) \mathscr{H}=$ $\phi^{-1}(\phi(\mathscr{H}))$. In particular, $\operatorname{Im}(\phi)=\phi(\mathscr{G})$ and $\operatorname{Ker}(\phi) \mathscr{H}$ are subgroupoids of $\mathscr{G}^{\prime}$ and $\mathscr{G}$, respectively.

(ii) If $\mathscr{H} \triangleleft \mathscr{G}$, then $\phi(\mathscr{H}) \triangleleft \phi(\mathscr{G})$.

(iii) ([29], Proposition 9) $\phi$ is an injective homomorphism, if and only if $\operatorname{Ker}(\phi)=\mathscr{G}_{0}$.

(iv) (The Correspondence Theorem for Groupoids) There exists a one-to-one correspondence between the sets $\mathscr{U}=\{\mathscr{H} \mid \mathscr{H}<\mathscr{G} \wedge \operatorname{Ker}(\phi) \subseteq \mathscr{H}\}$ and $\mathscr{B}=$ $\left\{\mathscr{H}^{\prime} \mid \mathscr{H}^{\prime}<\phi\left(\mathscr{G}^{\prime}\right)\right\}$. Moreover, this correspondence preserves normal subgroupoids.

Proof

(i) It is clear that $\phi(\mathscr{H}) \neq \varnothing$. Let $s, t \in \phi(\mathscr{H})$ and suppose that $\exists$ st. Then, $s=\phi(x), t=\phi(y)$ for some $x, y \in \mathscr{H}$. Since $\phi$ is strong, we have that $\exists x y$. Thus, $s t=\phi(x) \phi(y)=\phi(x y) \in \phi(\mathscr{H})$. Now, if $y \in \phi(\mathscr{H})$, then $\phi(x)=y$ for some $x \in \mathscr{H}$, and we have $y^{-1}=\phi(x)^{-1}=$ $\phi\left(x^{-1}\right) \in \phi(\mathscr{H})$.

Now, we check the equality $\operatorname{Ker}(\phi) \mathscr{H}=\phi^{-1}$ $(\phi(\mathscr{H}))$. If $g \in \phi^{-1}(\phi(\mathscr{H}))$, then there exists $h \in \mathscr{H}$ with $\phi(g)=\phi(h)$. Since $\phi$ is strong, we get that $\exists g h^{-1}$ and $g h^{-1} \in \operatorname{Ker}(\phi)$. Hence, $g=$ $\left(g h^{-1}\right) h \in \operatorname{Ker}(\phi) \mathscr{H}$. The other inclusion is clear.

(ii)-(iii) These are similar to the group case.

(iv) First, define the functions $\alpha: \mathscr{U} \longrightarrow \mathscr{B}$ by $\alpha(\mathscr{H})=\phi(\mathscr{H}) \quad$ for each $\mathscr{H} \in \mathscr{U}$, and $\beta: \mathscr{B} \longrightarrow \mathcal{U}$ by $\beta\left(\mathscr{H}^{\prime}\right)=\phi^{-1}\left(\mathscr{H}^{\prime}\right)$ for each $\mathscr{H}^{\prime} \in \mathscr{B}$. By (i) of Proposition 9 and (ii) of Proposition 10, it has that $\beta \circ \alpha=i d$ and $\alpha \circ \beta=i d_{\mathscr{B}}$. That is, $\alpha$ is a bijective function. The remaining proof follows from item (iii) of Proposition 9 and (ii) above.

Now, we use strong homomorphisms to extend to the groupoid context, a well-known result concerning the product of groups.

Proposition 11. Let $\mathscr{H}$ and $\mathscr{K}$ be subgroupoids of $\mathscr{G}$. If $\mathscr{K}$ is normal then

(i) $\mathscr{H} \mathscr{K}$ is a subgroupoid of $\mathscr{G}$

(ii) If $\mathscr{H}$ is normal, then $\mathscr{H} \mathscr{K}$ is a normal subgroupoid of $\mathscr{G}$

(iii) If $\mathscr{H}$ is wide, then $\mathscr{H} \cap \mathscr{K}$ is a normal subgroupoid of $\mathscr{H}$

Proof

(i) Consider the groupoid epimorphism $\phi: \mathscr{G} \ni g \longmapsto$ $g \mathscr{K} \in \mathscr{G} / \mathscr{K}$. Then, by the definition of $\mathscr{G} / \mathscr{K}$ the map $\phi$ is strong and $\operatorname{Ker}(\phi)=\mathscr{K}$. Thus, by (i) of Proposition 10 we get that $\mathscr{K} \mathscr{H}=\phi^{-1} \phi(\mathscr{H})$ is a subgroupoid of $\mathscr{G}$. Hence, the result follows from Proposition 7.

(ii) By the previous item, $\mathscr{H} \mathscr{K}$ is a subgroupoid of $\mathscr{G}$. Moreover, it is clear that $\mathscr{H} \mathscr{K}$ is wide. Let $g \in \mathscr{G}$ and $x \in \mathscr{H} \mathscr{K} \cap \mathscr{G}_{r(g)}$. Then, $x=h k$, with $h \in \mathscr{H}, k \in \mathscr{K}$, $d(h)=r(k)$ and $r(h)=d(k)=r(g)$. Thus, $\exists g^{-1} h k g$ and we have that

$$
\begin{aligned}
g^{-1} h k g & =g^{-1} h r(k) k g=g^{-1} h r(g) k g \\
& =\left(g^{-1} h g\right)\left(g^{-1} k g\right) \in \mathscr{H} \mathscr{K},
\end{aligned}
$$

that is, $\mathscr{H} \mathscr{K}$ is a normal subgroupoid of $\mathscr{G}$.

(iii) It is clear that $\mathscr{H} \cap \mathscr{K}$ is a wide subgroupoid of $\mathscr{H}$. Let $g \in \mathscr{H}$ and $h \in \mathscr{H} \cap \mathscr{K}$ with $r(h)=d(h)=$ $r(g)$. Then $\exists g^{-1} h g$ and by assumptions it follows that $g^{-1} h g \in \mathscr{H} \cap \mathscr{K}$.

Next we present the isomorphism theorems for groupoids.

Theorem 1 (the first isomorphism theorem). Let $\phi: \mathscr{G} \longrightarrow \mathscr{G}^{\prime}$ be a surjective strong groupoid homomorphism. Then there exists a strong isomorphism $\bar{\phi}: \mathscr{G} / \operatorname{Ker}(\phi) \longrightarrow \mathscr{G}^{\prime}$ such that $\phi=\bar{\phi} \circ j$, where $j$ is the canonical homomorphism of $\mathscr{G}$ onto $\mathscr{G} / \operatorname{Ker}(\phi)$.

Proof. Let $K=\operatorname{Ker}(\phi)$. We define $\bar{\phi}: \mathscr{G} / K \longrightarrow \mathscr{G}^{\prime}$ as $\bar{\phi}(x K)=\phi(x)$, for each $x K \in \mathscr{G} / K$. First of all, we show that $\bar{\phi}$ is a well defined function. Indeed, assume that $x K=y K$. Then $\exists y^{-1} x$ and $y^{-1} x \in K$. That is $\phi\left(y^{-1} x\right)=d(l)$ for some $l \in \mathscr{G}^{\prime}$, and then $\phi\left(y^{-1}\right) \phi(x)=d(l)$. Since $\phi$ is surjective, then $l=\phi(z)$, for some $z \in \mathscr{G}$. Multiplying the above equation by $\phi(y)$, we have that

$$
\begin{aligned}
\phi(y) \phi\left(y^{-1}\right) \phi(x) & =\phi(y) d(l) \\
& =\phi(y) d(\phi(z)) \\
& =\phi(y) \phi(d(z)) \\
& =\phi(y d(z)) .
\end{aligned}
$$

Then, $d(y)=d(z)$. So $\phi(y d(z))=\phi(y d(y))=\phi(y)$, whence $\phi(x)=\phi(y)$. Hence, $\bar{\phi}$ is well defined.

Now, note that $\bar{\phi}$ is a surjective strong homomorphism. Finally, we prove that $\bar{\phi}$ is injective. Indeed, assume that $\bar{\phi}(x K)=\bar{\phi}(y K)$, that is, $\phi(x)=\phi(y)$. Then, as $\phi$ is strong we have that $\phi\left(y^{-1} x\right) \in \mathscr{G}_{0}^{\prime}$. Thus, $y^{-1} x \in K$ and we have that $x K=y K$.

\section{Example 3}

(1) Consider the identity function $i_{\mathscr{G}}$ of the groupoid $\mathscr{G}$. Then, it is clear that $i_{\mathscr{G}}$ is a surjective strong homomorphism and $\operatorname{Ker}\left(i_{\mathscr{G}}\right)=\mathscr{G}_{0}$. Thus, by the first isomorphism theorem, we obtain that $\mathscr{G} / \mathscr{G}_{0} \cong \mathscr{G}$.

(2) Consider the function $\theta$ : Iso $(\mathscr{G}) \longrightarrow$ Iso $(\mathscr{G})$, defined by $\theta(g)=d(g)$ for all $g \in \operatorname{Iso}(\mathscr{G})$. For $g, h \in \operatorname{Iso}(\mathscr{G})$ suppose that $\exists g h$. Then, $d(g)=$ $r(h)=d(h), \exists d(g) d(h)$, and 
$\theta(g h)=d(g h)=d(h)=d(g) d(h)=\theta(g) \theta(h)$.

Now, let $g, h \in \operatorname{Iso}(\mathscr{G})$ such that $\exists \theta(g) \theta(h)$. Then, $\exists d(g) d(h)$ which implies that $d(g)=d(h)$ and since $d(h)=r(h)$ we obtain $\exists g h$. In conclusion, $\theta$ is a strong homomorphism, with $\operatorname{Ker}(\theta)=\operatorname{Iso}(\mathscr{G})$ and $\operatorname{Im}(\theta)=\mathscr{G}_{0}$. Whence, by the first isomorphism theorem, we obtain $\operatorname{Iso}(\mathscr{G}) / \operatorname{Iso}(\mathscr{G}) \cong \mathscr{G}_{0}$.

(3) Let $\mathscr{G}$ and $\mathscr{G}^{\prime}$ be a groupoids. The set $\mathscr{G} \times \mathscr{G}^{\prime}$ is a groupoid with the product defined by $\exists(x, y) \cdot(z, w)$ iff $\exists x \cdot z \wedge \exists y \cdot w$, and in this case $(x, y) \cdot(z, w)=(x \cdot z, y \cdot w)$. Moreover, note that $\left(\mathscr{G} \times \mathscr{G}^{\prime}\right)_{0}=\mathscr{G}_{0} \times \mathscr{G}_{0}^{\prime}$. If $\mathscr{H} \triangleleft \mathscr{G}$ and $\mathscr{K} \triangleleft \mathscr{G}^{\prime}$, then $\mathscr{H} \times$ $\mathscr{K} \triangleleft \mathscr{G} \times \mathscr{G}^{\prime} \quad$ and $\mathscr{G} \times\left(\mathscr{G}^{\prime} / \mathscr{H}\right) \times \mathscr{K} \cong(\mathscr{G} / \mathscr{H}) \times$ $\left(\mathscr{G}^{\prime} / \mathscr{K}\right)$. Indeed, it is clear that $\mathscr{H} \times \mathscr{K} \triangleleft \mathscr{G} \times \mathscr{G}^{\prime}$. For the second affirmation, define $\psi: \mathscr{G} \times \mathscr{G}^{\prime} \longrightarrow$ $(\mathscr{G} / \mathscr{H}) \times\left(\mathscr{G}^{\prime} / \mathscr{K}\right)$ by $\left(g, g^{\prime}\right) \longmapsto\left(g \mathscr{H}, g^{\prime} \mathscr{K}\right)$, and note that $\psi$ is a strong homomorphism. Moreover,

$$
\begin{aligned}
\operatorname{Ker}(\psi) & =\left\{\left(g, g^{\prime}\right) \in \mathscr{G} \times \mathscr{G}^{\prime} \mid \psi\left(g, g^{\prime}\right) \in(\mathscr{G} / \mathscr{H})_{0} \times\left(\mathscr{G}^{\prime} / \mathscr{K}\right)_{0}\right\} \\
& =\left\{\left(g, g^{\prime}\right) \in \mathscr{G} \times \mathscr{G}^{\prime} \mid\left(g \mathscr{H}, g^{\prime} \mathscr{K}\right) \in \mathscr{G}_{0} \mathscr{H} \times \mathscr{G}_{0}^{\prime} \mathscr{K}\right\} \\
& =\left\{\left(g, g^{\prime}\right) \in \mathscr{G} \times \mathscr{G}^{\prime} \mid\left(g, g^{\prime}\right) \in \mathscr{H} \times \mathscr{K}\right\} \\
& =\mathscr{H} \times \mathscr{K} .
\end{aligned}
$$

Thus, by the first isomorphism theorem the result follows.

Theorem 2 (the second isomorphism theorem). Let $\mathscr{G}$ be a groupoid, $\mathscr{M}$ a wide subgroupoid of $\mathscr{G}$, and $\mathscr{N}$ a normal subgroupoid of $\mathscr{G}$. Then, $\mathscr{M} \cap \mathscr{N} \triangleleft \mathscr{M}$ and

$$
\frac{\mathscr{M}}{\mathscr{M} \cap \mathcal{N}} \cong \frac{\mathscr{M N}}{\mathscr{N}}
$$

Proof. First, note that by (i) of Proposition $11, \mathscr{M} \mathcal{N}$ is a subgroupoid of $\mathscr{G}$. Moreover, since $\mathscr{N} \triangleleft \mathscr{G}$ we have $\mathscr{N} \triangleleft \mathscr{M} \mathscr{N}$. Also, it is clear that $\mathscr{M} \cap \mathscr{N} \triangleleft \mathscr{M}$.

We consider $\quad \psi: \mathscr{M} \longrightarrow(\mathscr{M} \mathscr{N} / \mathcal{N})$ given by $\psi(m)=m \mathscr{N}$ for all $m \in \mathscr{M}$. Then, it is clear that $\psi$ is a strong homomorphism. Furthermore, if $(m n) \mathcal{N} \in(\mathscr{M} \mathcal{N} / \mathcal{N})$, then $(m n) \mathcal{N}=m \mathcal{N}$. Thus, $\psi$ is surjective. Now,

$$
\begin{aligned}
\left(\frac{\mathscr{M} \mathscr{N}}{\mathscr{N}}\right)_{0} & =\left\{d(x \mathscr{N}) \mid x \mathscr{N} \in\left(\frac{\mathscr{M} \mathscr{N}}{\mathcal{N}}\right)\right\} \\
& =\{d(x) \mathscr{N} \mid x \in \mathscr{M} \mathcal{N}\} \\
& =\{d(x) \mathscr{N} \mid x=m n \wedge d(m)=r(n)\} \\
& =\{d(n) \mathscr{N} \mid n \in \mathscr{N}\} .
\end{aligned}
$$

On the other hand, $\operatorname{Ker}(\psi)=\{m \in \mathscr{M} \mid m \mathscr{N}=$ $d(n) \mathcal{N}$ for some $n \in \mathscr{N}\}=\mathscr{M} \cap \mathcal{N}$. Indeed, if $t \in \mathscr{M} \cap \mathcal{N}$, then $t \mathscr{N}=d\left(t^{-1}\right) \mathcal{N}$ and thus $t \in \operatorname{Ker}(\psi)$. For the other inclusion, if $m \in \operatorname{Ker}(\psi)$, then $m \in \mathscr{M}$ and $m \mathcal{N}=d(n) \mathcal{N}$ for some $n \in \mathcal{N}$. Thus, $\exists d(n) m$ and $d(n) m \in \mathcal{N}$. That is, $d(n)=r(m)$, and we have $d(n) m=r(m) m=m \in \mathcal{N}$. Finally, by Theorem 1 , we conclude that $(\mathscr{M} / \mathscr{M} \cap \mathscr{N}) \cong$ $(\mathscr{M} \mathscr{N} / \mathscr{N})$, as desired.

Remark 8. Given $M$ and $N$ as in Theorem 2, we saw in the proof of the same theorem that $\mathscr{N} \triangleleft \mathscr{M} \mathscr{N}$, which implies that
$\mathcal{N}_{0} \subseteq \mathscr{M}_{0}$. Indeed, let $e \in \mathcal{N}_{0}$. By Proposition 4 , there is $n \in N$ such that $e=r(n)$. Since $n=m^{\prime} n^{\prime}$ with $\left(m^{\prime}, n^{\prime}\right) \in(M \times$ $N) \cap \mathscr{G}^{2}$, then $e=r\left(m^{\prime}\right) \in \mathscr{M}_{0}$. Conversely, the condition $\mathcal{N}_{0} \subseteq \mathscr{M}_{0}$, clearly implies that $\mathscr{N} \triangleleft \mathscr{M} \mathscr{N}$. From this, we conclude that for $\mathscr{M}$ and $\mathscr{N}$ subgroupoids of $\mathscr{G}$, we have that $\mathscr{N} \triangleleft \mathscr{M} \mathscr{N}$, if and only if $\mathcal{N}_{0} \subseteq \mathscr{M}_{0}$.

Theorem 3 (the third isomorphism theorem). Let $\mathscr{G}$ be a groupoid, $\mathscr{H} \triangleleft \mathscr{G}$ and $\mathscr{K} \triangleleft \mathscr{G}$ with $\mathscr{K} \subseteq \mathscr{H}$. Then, $(\mathscr{H} / \mathscr{K}) \triangleleft$ $(\mathscr{G} / \mathscr{K})$ and

$$
\frac{\mathscr{G} \mid \mathscr{K}}{\mathscr{H} \mid \mathscr{K}} \cong\left(\frac{\mathscr{G}}{\mathscr{H}}\right) .
$$

Proof. Define $\varphi:(\mathscr{G} / \mathscr{K}) \longrightarrow(\mathscr{G} / \mathscr{H})$ by $\varphi(g \mathscr{K})=g \mathscr{H}$. First of all, we show that $\varphi$ is a well-defined function. Indeed, if $g \mathscr{K}=l \mathscr{K}$, then $\exists l^{-1} g$ and $l^{-1} g \in \mathscr{K}$. Since $\mathscr{K}<\mathscr{H}$, we have $l^{-1} g \in \mathscr{H}$, and hence $g \mathscr{H}=l \mathscr{H}$. Now,

$$
\begin{aligned}
\operatorname{Ker}(\varphi) & =\left\{g \mathscr{K} \mid g \mathscr{H} \in\left(\frac{\mathscr{G}}{\mathscr{H}}\right)_{0}\right\}=\left\{g \mathscr{K} \mid g \mathscr{H} \in \mathscr{G}_{0} \mathscr{H}\right\} \\
& =\{g \mathscr{K} \mid g \in \mathscr{H}\}=\left(\frac{\mathscr{H}}{\mathscr{K}}\right) .
\end{aligned}
$$

Thus, $\mathscr{H} / \mathscr{K} \triangleleft \mathscr{G} / \mathscr{K}$ and Theorem 1 implies that

$$
\frac{\mathscr{G} \mid \mathscr{K}}{\mathscr{H} \mid \mathscr{K}} \cong\left(\frac{\mathscr{G}}{\mathscr{H}}\right) .
$$

\section{Normal and Subnormal Series for Groupoids}

In this section, we present some applications of the isomorphism theorems of groupoids to normal and subnormal series. In particular, we show that the JordanHölder Theorem is also fulfilled in the context of 
groupoids. First, we introduce the following natural definitions.

Definition 10. Let $\mathscr{G}$ be a groupoid. Then,

(i) A subnormal series of a groupoid $\mathscr{G}$, is a chain of subgroupoids $\mathscr{G}^{(0)}=\mathscr{G}>\mathscr{G}^{(1)}>\cdots>\mathscr{G}^{(n)}$ such that $\mathscr{G}^{(i+1)}$ is normal in $\mathscr{G}^{(i)}$ for $0 \leq i \leq n$. The factors of the series are the quotient groupoids $\mathscr{G}^{(i)} / \mathscr{G}^{(i+1)}$. The length of the series is the number of strict inclusions. A subnormal series such that $\mathscr{G}^{(i)}$ is normal in $\mathscr{G}$ for all $i$, is called normal.

(ii) Let $\mathcal{S}: \mathscr{G}=\mathscr{G}^{(0)}>\mathscr{G}^{(1)}>\cdots>\mathscr{G}^{(n)}$ be a subnormal series. A one-step refinement of this series is any series of the form $\mathscr{G}=\mathscr{G}^{(0)}>\cdots>\mathscr{G}^{(i)}>N>$ $\mathscr{G}^{(i+1)}>\cdots>\mathscr{G}^{(n)}$ or $\mathscr{G}=\mathscr{G}^{(0)}>\cdots>\mathscr{G}^{(n)}>N$, where $\mathrm{N}$ is a normal subrgoupoid of $\mathscr{G}^{(i)}$ and $\mathscr{G}^{(i+1)}$ is normal in $\mathrm{N}$ (if $i<n)$ ). A refinement of a subnormal series $S$ is any subnormal series obtained from $S$ by a finite sequence of one-step refinements. A refinement of $S$ is called to be proper if it is larger than the length of $S$.

(iii) A subnormal series $\mathscr{G}=\mathscr{G}^{(0)}>\mathscr{G}^{(1)}>\cdots>\mathscr{G}^{(n)}=$ $\mathscr{G}_{0}$ is a composition series if each factor is simple; that is, its only normal subgroupoids are $\mathscr{G}$ and $\mathscr{G}_{0}$, and it is solvable if each factor is abelian.

Remark 9. It follows from (iv) of Proposition 10 that if $\mathcal{N}$ is a normal subgroupoid of a groupoid $\mathscr{G}$, every normal subgroupoid of $\mathscr{G} / \mathcal{N}$ is of the form $\mathscr{H} / \mathscr{N}$ where $\mathscr{H}$ is a normal subroupoid of $\mathscr{G}$, which contains $\mathscr{N}$. Thus, if $\mathscr{G} \neq \mathscr{N}$, then $\mathscr{G} / \mathscr{N}$ is simple, if and only if $\mathscr{N}$ is a maximal element in the set of all the normal subgroupoids $\mathscr{M}$ of $\mathscr{G}$, such that $\mathscr{M} \neq \mathscr{G}$.

Proposition 12. Let $\mathscr{G}$ be a groupoid. Then,

(i) If $\mathscr{G}$ is finite, then it has a composition series

(ii) Every refinement of a solvable series is a solvable series

(iii) A subnormal series is a composition series, if and only if it has no proper refinements

Proof

(i) Let $\mathscr{G}^{(1)}$ be a maximal normal subgroupoid of $\mathscr{G}$. Then, $\mathscr{G} / \mathscr{G}^{(1)}$ is simple by (iv) of Proposition 10. Let $\mathscr{G}^{(2)}$ be a maximal normal subgroupoid of $\mathscr{G}^{(1)}$, and so on. Now, since $\mathscr{G}$ is finite, this process must end with $\mathscr{G}^{(n)}=\mathscr{G}_{0}$. Thus, $\mathscr{G}=\mathscr{G}^{(0)}>\mathscr{G}^{(1)}>\cdots>$ $\mathscr{G}^{(n)}=\mathscr{G}_{0}$ is a composition series.

(ii) Here we use Remark 7 to observe that if $\mathscr{G}^{(i)} / \mathscr{G}^{(i+1)}$ is abelian and $\mathscr{G}^{(i+1)} \triangleleft \mathscr{H} \triangleleft \mathscr{G}^{(i)}$, then $\mathscr{H} / \mathscr{G}^{(i+1)}$ is abelian since it is a subgroupoid of $\mathscr{G}^{(i)} / \mathscr{G}^{(i+1)}$. Moreover, $\mathscr{G}^{(i)} / \mathscr{H}$ is abelian since it is isomorphic to $\left(\mathscr{G}^{(i)} / \mathscr{G}^{(i+1)}\right) /\left(\mathscr{H} / \mathscr{G}^{(i+1)}\right)$ by Theorem 3.

(iii) It follows from (iv) of Proposition 10 and that a subnormal series $\mathscr{G}=\mathscr{G}^{(0)}>\mathscr{G}^{(1)}>\cdots>\mathscr{G}^{(n)}=\mathscr{G}_{0}$ has a proper refinement, if and only if there is a subgroupoid $\mathscr{H}$ such that for some $i, \mathscr{G}^{(i+1)} \triangleleft$ $\mathscr{H} \triangleleft \mathscr{G}^{(i)}$ with $\mathscr{H}$ proper in $\mathscr{G}^{(i)}$ and $\mathscr{G}^{(i+1)}$ proper in $\mathscr{H}$.

Definition 11. Two subnormal series $\mathcal{S}$ and $\mathscr{T}$ of a groupoid $\mathscr{G}$ are equivalent, if there is a one-to-one correspondence between the nontrivial factors of $\mathcal{S}$ and the nontrivial factors of $\mathscr{T}$, such that the corresponding factors are isomorphic groupoids.

Lemma 3. If $\mathcal{S}$ is a composition series of a groupoid $\mathscr{G}$, then any refinement of $\mathcal{S}$ is equivalent to $\mathcal{S}$.

Proof. Let $\mathcal{S}: \mathscr{G}=\mathscr{G}^{(0)}>\mathscr{G}^{(1)}>\cdots>\mathscr{G}^{(n)}=\mathscr{G}_{0}$. By Proposition 12 (iii), $\mathcal{S}$ has no proper refinement. Thus, the only possible refinements of $\mathcal{S}$ are obtained by inserting additional copies of each $\mathscr{G}^{(i)}$. Whence, any refinement of $\mathcal{S}$ has exactly the same nontrivial factors as $\mathcal{S}$. Therefore, it is equivalent to $\mathcal{S}$.

Lemma 4 (Zassenhaus theorem for groupoids). Let $\mathscr{A}^{*}, \mathscr{A}, \mathscr{B}^{*}, \mathscr{B}$ be wide subgroupoids of a groupoid $\mathscr{G}$ such that

(i) $\mathscr{A}^{*}$ is normal in $\mathscr{A}$

(ii) $\mathscr{B}^{*}$ is normal in $\mathscr{B}$

Then, $\mathscr{A}^{*}(\mathscr{A} \cap \mathscr{B})$ and $\mathscr{B}^{*}(\mathscr{A} \cap \mathscr{B})$ are subgroupoids of $\mathscr{G}$ such that

(i) $\mathscr{A}^{*}\left(\mathscr{A} \cap \mathscr{B}^{*}\right)$ is a normal subgroupoid of $\mathscr{A}^{*}(\mathscr{A} \cap \mathscr{B})$

(ii) $\mathscr{B}^{*}\left(\mathscr{A}^{*} \cap \mathscr{B}\right)$ is a normal subgroupoid of $\mathscr{B}^{*}(\mathscr{A} \cap \mathscr{B})$

(iii) $\left(\mathscr{A}^{*}(\mathscr{A} \cap \mathscr{B}) / \mathscr{A}^{*}\left(\mathscr{A} \cap \mathscr{B}^{*}\right)\right) \cong$ $\left(\mathscr{B}^{*}(\mathscr{A} \cap \mathscr{B}) / \mathscr{B}^{*}\left(\mathscr{A}^{*} \cap \mathscr{B}\right)\right)$

Proof. (i) Since $\mathscr{B}^{*}$ is normal in $\mathscr{B}, \mathscr{A} \cap \mathscr{B}^{*}=$ $(\mathscr{A} \cap \mathscr{B}) \cap \mathscr{B}^{*}$ is a normal subgroupoid of $\mathscr{A} \cap \mathscr{B}$ thanks to (iii) of Proposition 11; similarly, $\mathscr{A}^{*} \cap \mathscr{B}$ is normal in $\mathscr{A} \cap \mathscr{B}$. Then, $\mathscr{D}=\left(\mathscr{A}^{*} \cap \mathscr{B}\right)\left(\mathscr{A} \cap \mathscr{B}^{*}\right)$ is a normal subgroupoid of $\mathscr{A} \cap \mathscr{B}$ by (ii) of Proposition 11. Also, by this same proposition, we have that $\mathscr{A}^{*}(\mathscr{A} \cap \mathscr{B})$ and $\mathscr{B}^{*}(\mathscr{A} \cap \mathscr{B})$ are subgroupoids of $\mathscr{A}$ and $\mathscr{B}$ respectively. Now, we define

$$
\tau: \mathscr{A}^{*}(\mathscr{A} \cap \mathscr{B}) \ni a c \longmapsto \tau(a c)=\mathscr{D} c \in\left(\frac{(\mathscr{A} \cap \mathscr{B})}{\mathscr{D}}\right),
$$

for all $a \in \mathscr{A}^{*}, c \in \mathscr{A} \cap \mathscr{B}$. The map $\tau$ is well defined since $a c=a_{1} c_{1}$ with $a, a_{1} \in \mathscr{A}^{*} ; c, c_{1} \in \mathscr{A} \cap \mathscr{B}$, implies that

$$
c_{1} c^{-1}=a_{1}^{-1} a \in(\mathscr{A} \cap \mathscr{B}) \cap \mathscr{A}^{*}=\mathscr{A}^{*} \cap \mathscr{B} \subseteq \mathscr{D},
$$

whence $\mathscr{D} c_{1}=\mathscr{D} c$. The map $\tau$ is clearly a strong epimorphism, and the equality $\operatorname{Ker}(\tau)=\mathscr{A}^{*}\left(\mathscr{A} \cap \mathscr{B}^{*}\right)$ is shown in an analogous way to the group case. 
Thus, Proposition 9 (iv) implies that $\mathscr{A}^{*}\left(\mathscr{A} \cap \mathscr{B}^{*}\right)$ is normal in $\mathscr{A}^{*}(\mathscr{A} \cap \mathscr{B})$, and by the first isomorphism theorem, we get $\mathscr{A}^{*}(\mathscr{A} \cap \mathscr{B}) / \mathscr{A}^{*}\left(\mathscr{A} \cap \mathscr{B}^{*}\right) \cong(\mathscr{A} \cap \mathscr{B}) / \mathscr{D}$.

A symmetric argument shows that $\mathscr{B}^{*}\left(\mathscr{B} \cap \mathscr{A}^{*}\right)$ is normal in $\mathscr{B}^{*}(\mathscr{A} \cap \mathscr{B})$ and $\left(\mathscr{B}^{*}(\mathscr{A} \cap \mathscr{B}) / \mathscr{B}^{*}\left(\mathscr{A}^{*} \cap \mathscr{B}\right)\right) \cong$ $((\mathscr{A} \cap \mathscr{B}) / \mathscr{D})$. Whence (iii) follows.

Proposition 13 (Schreier theorem for groupoids). Any two subnormal (resp. normal) series of a groupoid $\mathscr{G}$ have subnormal (resp. normal) refinement, which are equivalent.

Proof. It follows from Lemma 4, (ii) of Proposition 11, and Proposition 7 (1).

Proposition 14 (Jordan-Hölder theorem for groupoids). Any two composition series of a groupoid $\mathscr{G}$ are equivalent.

Proof. It follows from Proposition 13 and Lemma 3.

4.1. Some Remarks on the Equivalence between Inductive Groupoids and Inverse Semigroups. Recall that an inverse semigroup, is a semigroup $S$ such that for any $s \in S$ there is a unique $s^{*} \in S$ such that $s^{*}=s^{*} s s^{*}$ and $s=s s^{*} s$. Now, let $X$ be a set and consider the inverse semigroup

$\mathscr{G}(X)=\{f: A \longrightarrow B \mid A \subseteq X, B \subseteq X$ and $f$ is a bijection $\}$.

We recall the following.

Definition 12. Let $S$ be a semigroup. An action of $S$ on $X$ is a semigroup homomorphism $\phi: S \longrightarrow \mathscr{G}(X)$.

It follows from Theorem 4.2 of [23]) that partial actions of a group $G$ on $X$ are in one-to-one correspondence with actions of $E(G)$ on $X$, where $E(G)$ is the semigroup generated by the symbols $\{[g] \mid g \in G\}$ under the following relations: for $g, h \in G$,

$$
\begin{aligned}
{\left[g^{-1}\right][g][h] } & =\left[g^{-1}\right][g h], \\
{[g][h]\left[h^{-1}\right] } & =[g h]\left[h^{-1}\right], \\
{[g][1] } & =[g] .
\end{aligned}
$$

The semigroup $E(G)$ was introduced in [23], and it is called the Exel semigroup of .

Remark 10. Now, we present some facts about $E(G)$.

(1) The semigroup $E(G)$ is a monoid with $1_{E(G)}=[1]$.

(2) ([23], Proposition 2.5). For each $g \in G$ let $\gamma_{g}=$ $[g]\left[g^{-1}\right]$. Then, $\gamma_{g}$ is an idempotent of $E(G)$, each element $\alpha \in E(G)$ may be uniquely written (up to the order of the $s_{i}$ 's) as

$$
\alpha=\gamma_{s_{1}}, \gamma_{s_{2}}, \ldots, \gamma_{s_{n}}[g]
$$

for some $s_{1}, s_{2}, \ldots, s_{n}, g \in G$, with $g \neq s_{i} \neq s_{j} \neq g, i \neq j$ and $s_{i} \neq 1$, for $i \in\{1, \ldots, n\}$. From equation (25), it follows that any idempotent in $E(G)$ has the form $\gamma_{s_{1}} \gamma_{s_{2}} \ldots \gamma_{s_{n}}$ for some (uniquely) $s_{1}, s_{2}, \ldots, s_{n}, g \in G$.

(3) ([23], Theorem 3.4) The set $E(G)$ is an inverse semigroup. In particular, the idempotents of $E(G)$ commute (see [22], Theorem 3)).

Given an inverse semigroup $S$ and $s, t \in S$, one defines the restricted product

$$
s \cdot t \text { exists if and only if } s^{*} s=t t^{*} .
$$

It follows from Proposition 3.1.4 and Proposition 4.1.1 that $(S, \cdot, \leq)$ is an inductive groupoid (see [22], p. 108), where $\leq$ is the natural partial order defined on $S$. Then, by using the restricted product in $\mathscr{G}(X)$, we have that

$$
\mathscr{G}^{2}(X)=\{(f, g) \in \mathscr{G}(X) \times \mathscr{G}(X) \mid \operatorname{img}=\operatorname{dom} f\},
$$

and $\mathscr{G}(X)$ is a groupoid with the product given by composition of maps restricted to $\mathscr{G}^{2}(X)$. Moreover, $\mathscr{G}(X)_{0}=$ $\left\{\operatorname{id}_{A} \mid A \subseteq X\right\}$.

With respect to the semigroup $E(G)$, we have the following result.

Proposition 15. Let $\alpha=\gamma_{s_{1}} \gamma_{s_{2}} \ldots \gamma_{s_{n}}[g]$ and $\beta=\gamma_{t_{1}} \gamma_{t_{2}} \ldots$ $\gamma_{t_{m}}[l]$ where $s_{1}, s_{2}, \ldots, s_{n}, t_{1}, t_{2}, \ldots, t_{m}, g, l \in G$ are as in (25) of Remark 10. Then $\alpha \alpha^{*}=\beta^{*} \beta$, if and only if $\left\{s_{1}, s_{2}, \ldots\right.$, $\left.s_{n}, g\right\}=\left\{t_{1}, t_{2}, \ldots, t_{n}, l^{-1}\right\}$.

Proof. We have that $\alpha \alpha^{*}=\gamma_{s_{1}}, \gamma_{s_{2}}, \ldots, \gamma_{s_{n}}[g]\left[g^{-1}\right] \gamma_{s_{1}}$, $\gamma_{s_{2}}, \ldots, \gamma_{s_{n}}=\gamma_{s_{1}}, \gamma_{s_{2}}, \ldots, \gamma_{s_{n}}, \gamma_{g}$. Then,

$$
\begin{aligned}
\alpha \alpha^{*}=\beta^{*} \beta & \Longleftrightarrow \gamma_{s_{1}}, \gamma_{s_{2}}, \ldots, \gamma_{s_{n}}, \gamma_{g}=\gamma_{t_{1}}, \gamma_{t_{2}}, \ldots, \gamma_{t_{m}}, \gamma_{t^{-1}} \\
& \Longleftrightarrow\left\{s_{1}, s_{2}, \ldots, s_{n}, g\right\}=\left\{t_{1}, t_{2}, \ldots, t_{n}, l^{-1}\right\},
\end{aligned}
$$

where the last equivalence follows from (2) of Remark 10.

Using the restricted product to provide $E(G)$ with a groupoid structure we get by Proposition 15 that,

$$
\begin{aligned}
E(G)^{2} & =\left\{\left(\gamma_{s_{1}}, \ldots, \gamma_{s_{n}}[g], \gamma_{t_{1}}, \ldots, \gamma_{t_{m}}[l]\right) \mid\left\{s_{1}, \ldots, s_{n}, g\right\}\right. \\
& \left.=\left\{t_{1}, \ldots, t_{n}, l^{-1}\right\}\right\},
\end{aligned}
$$

and $E(G)_{0}=\left\{\gamma_{s_{1}} \gamma_{s_{2}} \cdots \gamma_{s_{n}} \mid s_{i} \neq s_{j}, i \neq j, n \in \mathbb{N}\right\}$.

From [7], it follows that a global action $\beta$ of a groupoid $\mathscr{G}$ on $X$ is a family of bijections $\beta=\left\{\beta_{g}: X_{g^{-1}} \longrightarrow X_{g} \mid g \in \mathscr{G}\right\}$ such that

(i) $X=\% \cup_{e \in \mathscr{G}_{0}} X_{e}$

(ii) $\beta_{e}=\mathrm{id}_{X_{e}}$, for all $e \in \mathscr{G}_{0}$

(iii) $\beta_{g} \circ \beta_{h}=\beta_{g h}$, for all $(g, h) \in \mathscr{G}^{2}$

Then according to ([7], Proposition 10), global actions of $\mathscr{G}$ on $X$ correspond to groupoid homomorphism $\mathscr{G} \longrightarrow \mathscr{G}(X)$. On the other hand, in the case when $\mathscr{G}$ is a group we obtain the definition of a partial group action on a set (see [2], Definition 1.2) 
If $\mathscr{G}$ is an inductive groupoid, then Proposition 4.1.7 of [22]) implies that $(\mathscr{G}, \otimes)$ is an inverse semigroup, where $\otimes$ denotes the pseudo product defined on $\mathscr{G}$ (see [22], p. 112).

Then, we have the following.

Proposition 16. For every group $G$ and any set $X$, there is $a$ one-to-one correspondence between
(1) Partial actions of $G$ on $X$
(2) Unital semigroup actions of $E(G)$ on $X$
(3) Groupoid homomorphisms $E(G) \longrightarrow \mathscr{G}(X)$
(4) Groupoid actions of $E(G)$ on $X$

Proof. We have already observed that there is a one-to-one correspondence between partial actions of $G$ on $X$ and semigroup actions of $E(G)$ on $X$, and between groupoid homomorphisms $E(G) \longrightarrow \mathscr{G}(X)$, and global actions of $G$ on $X$. Moreover, given a semigroup action $\phi: E(G) \longrightarrow$ $\mathscr{G}(X)$, then let $\beta_{\alpha}=\phi(\alpha)$ and $X_{\alpha}=\operatorname{im}(\phi(\alpha))$. Since $\phi([1])=\mathrm{id}_{X}$, one has that the family $\beta=\left\{\beta_{\alpha}\right.$ : $\left.X_{\alpha^{-1}} \longrightarrow X_{\alpha}\right\}_{\alpha \in E(G)}$ is a global action of $E(G)$ on $X$. Conversely, given a global action $\beta=\left\{\beta_{\alpha}: X_{\alpha^{-1}} \longrightarrow X_{\alpha}\right\}_{\alpha \in E(G)}$ of $E(G)$ on $X$, let $\varphi: E(G) \ni \alpha \longmapsto \beta_{\alpha} \in \mathscr{G}(X)$. Then, $\varphi$ is an action of $E(G)$ on $X$. Indeed, if $\alpha, \beta \in E(G)$, then by Proposition 4.1.7, we have that $\alpha \gamma=\alpha \otimes \gamma$ and $\phi(\alpha \gamma)=$ $\phi(\alpha \otimes \gamma)=\phi(\alpha) \otimes \phi(\gamma)=\phi(\alpha) \circ \phi(\gamma)$, as desired.

\section{Data Availability}

No data were used to support this study.

\section{Conflicts of Interest}

The authors declare that there are no conflicts of interest regarding the publication of this article.

\section{References}

[1] H. Brandt, "Über eine verallgemeinerung des gruppenbegriffes," Mathematische Annalen, vol. 96, no. 1, pp. 360-366, 1926.

[2] C. Ehresmann, "Oeuvres complétes. Parties I.1, I.2," in Topologie algéBrique et géométrie différentielle, Imprimerie Evrard, Amiens, France, 1950.

[3] R. Brown, "From groups to groupoids: a brief survey," Bulletin of the London Mathematical Society, vol. 19, no. 2, pp. 113134, 1987.

[4] G. Ivan, "Strong morphisms of groupoids," Balkan Journal of Geometry and Its Applications, vol. 4, no. 1, pp. 91-102, 1999.

[5] G. Ivan, "Algebraic constructions of brandt groupoids," in Proceedings of the Algebra Symposium, Babes-Bolyai University Cluj, Cluj-Napoca, Romania, November 2002.

[6] N. D. Gilbert, "Actions and expansions of ordered groupoids," Journal of Pure and Applied Algebra, vol. 198, no. 1-3, pp. 175-195, 2005.

[7] P. Nystedt, "Partial category actions on sets and topological spaces," Communications in Algebra, vol. 46, no. 2, pp. 671-683, 2018.

[8] F. Abadie, "On partial actions and groupoids," Proceedings of the American Mathematical Society, vol. 132, no. 4, pp. 1037-1048, 2004.
[9] F. Abadie, "Partial actions of discrete groups and related structures," Publicaciones Matemáticas del Uruguay, vol. 10, pp. 1-9, 2005.

[10] D. Bagio, "Partial actions of inductive groupoids on rings," International Journal of Mathematics, Game Theory, and Algebra, vol. 20, pp. 33-47, 2011.

[11] D. Bagio, D. Flores, and A. Paques, "Partial actions of ordered groupoids on rings," Journal of Algebra and Its Applications, vol. 09, no. 3, pp. 501-517, 2010.

[12] D. Bagio and A. Paques, "Partial groupoid actions: globalization, Morita theory, and galois theory," Communications in Algebra, vol. 40, no. 10, pp. 3658-3678, 2012.

[13] D. Bagio and H. Pinedo, "On the separability of the partial skew groupoid ring," São Paulo Journal of Mathematical Sciences, vol. 11, no. 2, pp. 370-384, 2017.

[14] P. Nystedt, J. Ö.sted, and H. Pinedo, "Artinian and noetherian partial skew groupoid rings," Journal of Algebra, vol. 503, pp. 433-452, 2018.

[15] P. Nystedt, J. Ö.sted, and H. Pinedo, "Epsilon strongly groupoid rings: the picard inverse category and cohomology," Glasgow Mathematical Journal, vol. 62, no. 1, pp. 233-259, 2020.

[16] A. Paques and D. Flôres, "Duality for groupoid (co)actions," Communications in Algebra, vol. 42, no. 2, pp. 637-663, 2014.

[17] W. Cortes and T. Tamusiunas, "A characterisation for a groupoid galois extension using partial isomorphisms," Bulletin of the Australian Mathematical Society, vol. 96, no. 1, pp. 59-68, 2017.

[18] A. Paques and T. Tamusiunas, "A Galois-Grothendieck type correspondence for groupoid actions," Algebra and Discrete mathematics, vol. 17, no. 1, pp. 80-97, 2014.

[19] A. Paques and T. Tamusiunas, "The Galois correspondence theorem for groupoid actions," Journal of Algebra, vol. 509, pp. 105-123, 2018

[20] D. Bagio and H. Pinedo, "Globalization of partial actions of groupoids on nonunital rings," Journal of Algebra and Its Applications, vol. 15, no. 5, Article ID 1650096, 2016.

[21] V. Marín and H. Pinedo, "Partial groupoid actions on $R$ categories: globalization and the smash product," Journal of Algebra and Its Applications, Article ID 2050083, 2020.

[22] M. V. Lawson, Inverse Semigroups. The Theory of Partial Symmetries, World Scientific Pub. Co., Singapore, 1995.

[23] R. Exel, "Partial actions of groups and actions of inverse semigroups," Proceedings of the American Mathematical Society, vol. 126, no. 12, pp. 3481-3495, 1998.

[24] J. Renault, "A groupoid approach to $C^{*}$-algebras," Lecture Notes in Mathematics, vol. 793, no. 2, 1980.

[25] D. Flôres, Acão de grupoides sobre álgebras: teoremas de estructura, Ph.D. thesis, UFRGS, Porto Alegre, Brazil, 2011.

[26] A. Ramsay, "Virtual groups and group actions," Advances in Mathematics, vol. 6, no. 3, pp. 253-322, 1971.

[27] J. Westman, Groupoid Theory in Algebra, Topology and Analysis, University of California at Irvine, Irvine, CA, USA, 1971.

[28] H. Myrnouri and M. Amini, "Decomposable abelian groupoids," International Mathematical Forum, vol. 5, pp. 2371-2380, 2010.

[29] P. Stachura, "Short and biased introduction to groupoids," Journal of Knot Theory and Its Ramifications, vol. 27, no. 7, Article ID 1841010, 2018. 